\title{
Flujo de Carbono Orgánico Total (COT) en una cuenca andina: caso subcuenca Río Las Piedras
}

\author{
María Cristina Ordóñez Díaz \\ Isabel Bravo Realpe \\ Apolinar Figueroa Casas ${ }^{* * *}$
}

Recibido: 18/07/2013 • Aceptado: 11/10/2013

\begin{abstract}
Resumen
El estudio se realizó para calcular el flujo de carbono transportado por la cuenca del río Las Piedras, típica representativa de los Andes tropicales suramericanos, reservorio estratégico del recurso hídrico. Se desarrolló una investigación observacional durante 20 meses sobre el recurso hídrico, mediante un diseño completamente aleatorio, estratificado por altura (msnm), analizando el contenido de COT, las propiedades físico-químicas del agua y variables hidrometeorológicas (caudal y variación estacional). La concentración promedio de COT aportada principalmente desde la zona alta fue $9 \mathrm{mgL}^{-1}$. Se transporta en promedio un flujo de 2003,5 Kg.dia-1 de COT, asociado al caudal y la estacionalidad húmeda. El análisis en relación con variables físico-químicas permite concluir que el río disuelve $\mathrm{CO}_{2}$ en sus aguas Este estudio contribuye al análisis del comportamiento de los ríos andinos y su aporte al ciclo global del carbono.
\end{abstract}

Palabras clave: calidad del agua, variación estacional, ciclo del carbono.

Bióloga. PhD (C) Ciencias Ambientales. Investigadora Grupo de Estudios Ambientales. Universidad del Cauca. Calle 2 \# 1A- 25 Urbanización Caldas. Popayán-Cauca. Colombia.mcordonez@unicauca.edu.co

** MSc. Quimica. Docente Universidad del Cauca. Grupo de Agroquímica. Cra 2 A \# 3N-111 Tulcán PopayánCauca. Popayán-Cauca. Colombia. ibravo@unicauca.edu.co

*** PhD. Docente. Investigadora Grupo de Estudios Ambientales. Universidad del Cauca. Calle 2 \# 1A- 25 Urbanización Caldas. Popayán-Cauca. Colombia. apolinar@unicauca.edu.co 


\title{
Total Organic Carbon (TOC) flow in an andean watershed: Las Piedras River sub-watershed case
}

\begin{abstract}
The study was conducted in order to calculate the flow of carbon carried by the Las Piedras river watershed, typical and representative of the South American tropical Andes, strategic reservoir of the hydrological resource. An observational research was performed over 20 months on the hydrological resource by means of a completely random design, stratified by altitude (masl), analyzing the TOC content, the physicochemical properties of the water and hydro-meteorological variables (discharge and seasonal variation). The average concentration of TOC mainly contributed from the high zone was 9 mg.L-1. A TOC flow of $2003.5 \mathrm{Kg}$.day-1 associated with the discharge and the humid seasonality is carried in average. The analysis related to physicochemical variables allows concluding that the river dissolves $\mathrm{CO} 2$ in its waters. This study supplements the behavioral analysis of the Andean rivers and their contribution to the global carbon cycle.
\end{abstract}

Key words: water quality, seasonal variation, carbon cycle. 


\section{INTRODUCCIÓN}

Los ríos son el mayor conducto para la transferencia lateral de carbono de los continentes a los océanos, y funcionan como un conector del ciclo del carbono global, que transfieren al año entre 0,8 y $1,33 \mathrm{Pg}$ [1]. El dióxido de carbono $\left(\mathrm{CO}_{2}\right)$ puede ser transportado longitudinalmente por las aguas superficiales, y parcialmente transferido a la atmósfera en proporciones que dependen de la velocidad del flujo, de la diferencia de la presión parcial de $\mathrm{CO}_{2}\left(\mathrm{pCO}_{2}\right)$ y del coeficiente de intercambio de gases $(\mathrm{K})$. Los flujos fluviales de carbono (C) son sensibles a los cambios regionales y mundiales debido a que se ven afectados por procesos físicos, bióticos y antrópicos [2], controlados por la hidrología regional, la geología, el uso de la tierra, la físico-química de los suelos y el clima. Además, las actividades antropogénicas han aumentado dramáticamente las cargas de nutrientes a los ríos con fertilizantes orgánicos e inorgánicos. Se ha asociado al uso del suelo agrícola el deterioro de recurso hídrico por el flujo de sedimentos y carga de nutrientes transportados hacia los cuerpos de agua, afectando la calidad de la misma por efecto indirecto de los nutrientes, como son la eutrofización y cambios físico-químicos en el agua [3]. El aumento de la contaminación agrícola hacia las aguas superficiales genera preocupación considerable. Este interés y análisis es especialmente pertinente en cuencas sometidas a procesos de agricultura intensiva, donde se experimenta apreciable disminución de especies acuáticas, asociada a la presencia en el agua de niveles tóxicos de algunos elementos. Las principales fuentes de contaminación incluyen escorrentía agrícola y urbana, descargas de minas abandonadas y fuentes puntuales, que generalmente ocupan partes altas, medias y bajas de las cuencas andinas [4].

El transporte de carbono orgánico total (COT) a los océanos es un componente integral del presupuesto global de carbono y puede representar un valor importante en la retención del $\mathrm{CO}_{2}$ de la atmósfera. Sin embargo, el origen geológico de la roca, las características edafológicas de los suelos, constantes cambios en el uso de la tierra, actividades socioculturales y la relación entre la exportación de carbono y el clima son interrogantes importantes que han sido poco dilucidados para ecosistemas andinos.

Existen varios estudios de importancia sobre esta temática de interés y actualidad, realizados en cuencas hidrográficas, donde se han modelado flujos de C [1], así como su balance y emisión de $\mathrm{CO}_{2}[5]$. Entre ellos se destacan los de Butman and Raymod [6] quienes evaluaron las estimaciones actuales de la liberación de $\mathrm{CO}_{2}$ en sistemas de agua dulce, afirmando que están entre 0,7 a 3,3 $\mathrm{Pg} \mathrm{C}$ año $^{-1}$. Adicionalmente, se ha caracterizado la relación del C con factores como la topografía y la variabilidad climática [7]. En distintas localidades se han realizado estimaciones globales de COT en ríos $[8,9]$. 
Estos referentes permiten evidenciar que los esfuerzos mundiales se centran en entender los presupuestos regionales de carbono (las estimaciones de las fuentes, sumideros y transporte de $\mathrm{CO}_{2}$ ), y la contabilidad del carbono a escala local, para apoyar la gestión del carbono en el futuro. Sin embargo, a pesar de ser el carbono orgánico (CO) catalogado como la causa más común de deterioro de la calidad del agua [10], y como un elemento que modifica la influencia y consecuencia de otros productos químicos y procesos en los lagos y ríos, aún no es considerado un importante criterio de gestión, y los datos relevantes necesarios para su estudio en los sistemas afectados son escasos.

Esta investigación plantea que el cambio en el uso del suelo puede ser identificado como un acelerador de la exportación de carbono. La cuenca del río Las Piedras abastecedora del recurso hídrico para la ciudad de Popayán está siendo afectada por vertimientos y actividades agropecuarias (cultivos de papa, ganadería y criaderos de alevinos) con procesos de deforestación y pérdida significativa del suelo productivo, actividades que inciden en la calidad del recurso hídrico [4]. Las principales demandas de agua en esta cuenca requieren un líquido de excelente calidad, porque abastecen consumo humano local y de la población de Popayán y la piscifactoría de truchas. La dinámica de los ciclos biogeoquímicos de carbono en esta cuenca resultan de vital importancia, y cualquier perturbación antropogénica se verá reflejada en la calidad del agua. En este contexto, estudiar el comportamiento del contenido de $\mathrm{CO}$ en relación con propiedades fisicoquímicas del agua y variables climáticas contribuye a la estimación del flujo fluvial de carbono y su relación con procesos naturales y antropogénicos que impactan y alteran el ciclo natural del carbono.

\section{MATERIALES Y MÉTODO}

\section{1. Área de estudio}

El estudio se realizó en la cuenca del río las Piedras, típica representativa de los Andes ecuatoriales intertropicales suramericanos por sus características fisiográficas y por los servicios ecosistémicos que provee. Está ubicada en las coordenadas $2^{\circ} 21^{\prime} 35$ ” N y $76^{\circ} 33^{\prime} 10^{\prime}$ O $\mathrm{O}$, con extensión de $58 \mathrm{Km}^{2}$. La temperatura promedio media mensual varía entre los $10,4{ }^{\circ} \mathrm{C}$ y $18,4{ }^{\circ} \mathrm{C}$ [4]. El rango altitudinal de la cuenca está comprendido entre 1980 y $3820 \mathrm{msnm}$; corresponde a las formaciones de bosque subandino y bosque andino. El relieve es montañoso, con pendientes fuertes, largas y rectilíneas que tienen entre $35-98 \%$ en la zona alta, $16-35 \%$ en la zona media y de 3-15\% en la zona baja. Los suelos están formados principalmente a partir de cenizas volcánicas, con textura media franco-arcillosa poco estructurada y bien drenada; poseen acidez fuerte con $\mathrm{pH}$ entre 4,9 y 5,0, con alta saturación de aluminio y bajas cantidades de 
calcio, magnesio y fósforo [11]. El suelo se dedica principalmente a la proteccion de coberturas boscosas con un 27,9 \% (1.848,9 ha), a ganadería con un 22,9\% (pastizales) (1.523,1 ha), y agricultura con un $3,9 \%$ (256,9 ha) (figura 1$)$.

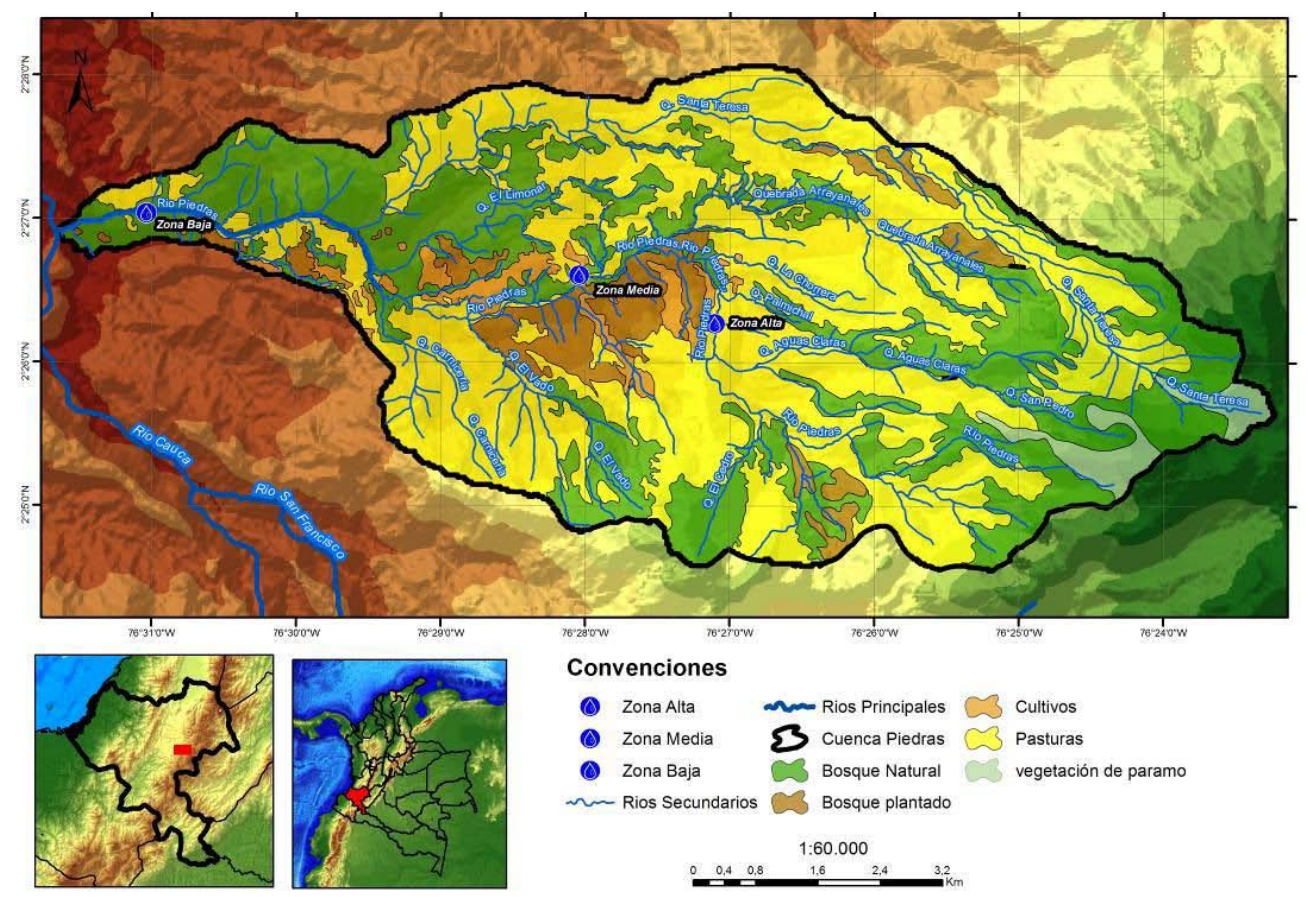

Figura 1. Ubicación de la cuenca del río Las Piedras, en el departamento del Cauca-Colombia. Fuente: elaboración propia

\subsection{Diseño experimental}

Se utilizó un diseño completamente aleatorio estratificado por altura sobre el nivel del mar; las unidades experimentales se seleccionaron considerando tres zonas: alta $(<2600$ $\mathrm{msnm})$, media (2300-2600) y baja $(<2300 \mathrm{msnm})$, en un tramo de $10 \mathrm{~km}$ a lo largo del cauce principal del río. Las muestras fueron colectadas en cada zona de forma puntual y mensual, mediante un recorrido en zigzag en un área aproximada de $25 \mathrm{~m}^{2}$ en un cauce ancho y turbulento; se tomaron 25 submuestras para formar la correspondiente muestra compuesta de 1L, para un total de 171 muestras compuestas para el estudio, durante un período de 20 meses de monitoreo (2009-2011). Se utilizaron recipientes de polietileno de alta densidad, previamente lavados con mezcla ácida y agua desionizada. Las muestras compuestas fueron tomadas con tres réplicas, para realizarle los análisis descritos en la tabla 1 [12]. 
Tabla 1. Evaluación de las propiedades físicas y químicas del agua.

\begin{tabular}{|c|c|c|c|}
\hline Parámetro & Técnica analítica-Instrumento & Precisión & Método \\
\hline $\mathrm{pH}$ & Electrometría & $\pm 0,01$ & $\mathrm{SM} 4500 \mathrm{H}$ \\
\hline Alcalinidad total $\left(\mathrm{mgL}^{-1}\right)$ & Titulometría & & SM 2320B \\
\hline Nitrógeno total $\left(\mathrm{mg} \mathrm{L}^{-1}\right)$ & Kjeldajh & $\pm 0,003$ & $\begin{array}{l}4500 \mathrm{~N}_{\text {org }} \mathrm{B} \\
\text { ISO } 5663 / 840\end{array}$ \\
\hline Oxígeno disuelto (mg L-1) & $\begin{array}{l}\text { Electrodo de membrana Multi-pará- } \\
\text { metro HACH HQ30d portátil }\end{array}$ & $\pm 0,1$ & SM 4500-O C \\
\hline Sólidos disueltos totales (mg L-1) & $\begin{array}{l}\text { Multi-parámetro } \mathrm{HACH} \text { HQ30d } \\
\text { portátil }\end{array}$ & $\pm 0,5 \%$ & SM 2540D \\
\hline Conductividad eléctrica $(\mu \mathrm{S} \mathrm{cm}-1)$ & $\begin{array}{l}\text { Electrometría Multi - parámetro } \\
\text { HACH HQ30d portátil }\end{array}$ & $\pm 0,5 \%$ & SM2320B \\
\hline Carbono orgánico total (mg L-1) & $\begin{array}{l}\text { Combustión-Infrarrojo Analizador } \\
\text { carbono Shimadzu TOC-VSCN }\end{array}$ & 0,01 & SM5310B \\
\hline Temperatura hídrica $\left({ }^{\circ} \mathrm{C}\right)$ & $\begin{array}{l}\text { Termométrico Multi-parámetro } \\
\text { HACH HQ30d portátil }\end{array}$ & $\pm 0,3^{\circ} \mathrm{C}$ & SM2550 B \\
\hline
\end{tabular}

Fuente: elaboración propia

\subsection{Determinación de parámetros hidrometeorológicos}

Se recopilaron registros diarios de pluviosidad y temperatura ambiente durante 20 meses, tomados de las estaciones de la cuenca río Las Piedras y Universidad del Cauca-GEA, ubicadas en la zona alta y baja, respectivamente. Adicionalmente se asoció la información mensual a la variabilidad intraanual clasificando en época seca y época húmeda. Para el análisis de caudales, se utiliza la curva de calibración de caudal frente a la altura, y registros de caudales para las tres zonas [13].

\subsection{Tratamiento estadístico de los resultados}

Para el procesamiento estadístico se empleó el programa SPSS versión 13,0, y se hizo un análisis de distribución y exploratorio de datos con aplicación de pruebas específicas para evaluar la dependencia y diferencias significativas de las variables, complementadas con análisis multivariados para integrar los parámetros de interés.

\section{RESULTADOS Y DISCUSIÓN}

\subsection{Carbono orgánico total}

La concentración COT en el tramo del río analizado de $10 \mathrm{~km}$ durante el período 
estudiado tiene en promedio $9 \mathrm{mg} \mathrm{L}^{-1}$, un valor máximo de $16,12 \mathrm{mg} \mathrm{L}^{-1}$ y un mínimo de $1,68 \mathrm{mg} \mathrm{L}^{-1}$ (figura 2), cercanos con los reportados en África $3.5 \mathrm{mg} / 1$ to $10.8 \mathrm{mg} / 1$. [14]. Estos valores están dentro del rango reportado para estudios realizados en aguas naturales $\left(0,5\right.$ - $\left.100 \mathrm{mg} \mathrm{L}^{-1}\right)$ [15]. No se encontraron diferencias longitudinales en las concentraciones de COT; por lo tanto, se deduce que el mayor aporte es realizado por la parte alta de la cuenca que cubre $28,9 \%$ del área.

La comparación de los flujos promedio de COT para cada una de las 3 zonas registra que la zona baja tiene el mayor flujo de COT con 2003,5 $\mathrm{kg}$ día $^{-1}$, seguido por la zona media $\left(1776,8 \mathrm{~kg} \mathrm{día}^{-1}\right)$ y alta $\left(1640,5 \mathrm{~kg} \mathrm{día}^{-1}\right)$. Al contrastar estos resultados con el caudal, la variación estacional intranual y la concentración de carbono (correlación de Spearman), se observa que existe correlación directa entre el flujo de COT y el caudal $\left(\mathrm{r}_{\mathrm{s}}=0,817^{* *}\right)$, el cual, a su vez, se encuentra influenciado por la variación estacional, donde se demuestra que el tiempo húmedo tiene relación directa con el caudal $\left(\mathrm{r}_{\mathrm{s}}=0,679^{* *}\right)$, influenciado por las precipitaciones características de la estacionalidad húmeda en la zona. Por el contrario, se observa una correlación negativa entre el caudal y la concentración de carbono al comparar los meses húmedos y secos (figura 3): a mayor caudal se presenta una menor concentración de C, y viceversa. Por lo anterior, se puede deducir que la variación longitudinal del flujo de carbono depende principalmente del caudal (Q) de cada zona, siendo la zona baja la que tiene el mayor flujo de COT, al poseer la mayor área y velocidad de corriente.

El comportamiento temporal del flujo de carbono durante los 20 meses de estudio, en relación con el caudal y la concentración de carbono, muestra que los meses 19-20 (noviembre y diciembre de 2011) registran los flujos más altos, correspondientes a los mayores caudales registrados; estos son catalogados como meses húmedos (figura 4). Los meses 16-17-18 (agosto, septiembre y octubre de 2011), a su vez, reportan el menor flujo de carbono, pues tienen los menores caudales. No se observa relación del flujo de COT con la concentración de carbono en cada mes, a pesar de que exista correlación positiva $\left(r_{\mathrm{s}}=0,492^{* *}\right)$. Por lo tanto, se deduce que el flujo de carbono en la cuenca depende principalmente de la variación en el caudal y la estacionalidad de la cuenca, tal como se enunció anteriormente con las correlaciones encontradas.

\subsection{Carbono orgánico total y variables fisicoquímicas}

$\mathrm{Al}$ analizar la influencia de la temporalidad sobre las variables analizadas en el agua del río (20 meses), se observan influencia temporal $(p=0,00)$ sobre las variables COT, $\mathrm{pH}$ (7,49 \pm 0.33$)$, nitrógeno total (NT), alcalinidad $(30,20 \pm$ y 8,12), sólidos disueltos totales (SDT) $(29,55 \pm 4,5)$, conductividad eléctrica (CE) $(62,94 \pm 9,4)$, oxígeno disuelto (OD) $(7,69 \pm 0,16)$, temperatura ambiental $\mathrm{T}\left({ }^{\circ} \mathrm{C}\right)$, y temperatura del agua $\left({ }^{\circ} \mathrm{C}\right)$. La época húmeda expresa que existe influencia significativa en los valores de NT, SDT, CE ( $p=0,00)$; 
esto debido posiblemente a la intensidad de lluvia (tabla 2) que genera escurrimiento superficial impactando sobre la degradación del suelo que, por erosión hídrica, afecta la cohesión de partículas, y genera pérdida de suelo; en consecuencia, un incremento en el transporte de SDT en el río y de iones en el agua, que produce incremento en la CE. Estas alteraciones en el agua del río pueden afectar la calidad física-química del agua, similar a lo presentado por Wu et al. [16], en una cuenca de China.

Tabla 2. Correlación de Spearman

\begin{tabular}{|c|c|c|c|c|c|c|c|c|}
\hline & & & $p H$ & $\begin{array}{c}N T \\
(m g L-1)\end{array}$ & $\begin{array}{c}\text { Alcalinidad } \\
(m g L-1)\end{array}$ & $\begin{array}{c}S D T \\
(m g L-1)\end{array}$ & $\begin{array}{c}C E \\
(m g L-1)\end{array}$ & $\begin{array}{c}O D \\
(m g L-1)\end{array}$ \\
\hline & & $\begin{array}{l}\text { Coeficiente de } \\
\text { correlación }\end{array}$ & $.379^{* *}$ & $.439^{* *}$ & -.153 & .102 & .093 & $-.237^{* *}$ \\
\hline & COT (mg L-1) & Sig. (bilateral) & .000 & .000 & .059 & .183 & .225 & .002 \\
\hline $\mathrm{Rho} \quad \mathrm{de}$ & & $\mathrm{N}$ & 171 & 156 & 153 & 171 & 171 & 171 \\
\hline Spearman & & $\begin{array}{l}\text { Coeficiente de } \\
\text { correlación }\end{array}$ & .104 & $-.338^{* *}$ & $-.247^{* *}$ & $-.636^{* *}$ & $-.621^{* *}$ & -.018 \\
\hline & $\begin{array}{l}\text { Precipitacion } \\
(\mathrm{mm})\end{array}$ & Sig. (bilateral) & .178 & .000 & .002 & .000 & .000 & .813 \\
\hline & & $\mathrm{N}$ & 171 & 156 & 153 & 171 & 171 & 171 \\
\hline
\end{tabular}

** Significativa a nivel 0.05 .

Fuente: elaboración propia

Las concentraciones de material orgánico y sedimentos exportados por los ríos andinos pueden incrementar con la magnitud de los eventos de lluvias frecuentes que incrementan los deslizamientos de tierra en zonas de pendiente, y tienen importantes implicaciones para la comprensión de geoquímica de los ríos. A pesar de que el COT no se vio influenciado significativamente por el régimen de lluvias, en la mayoría de los ríos de alta montaña, la mayor parte de $\mathrm{C}$ y $\mathrm{N}$ que entra al sistema se produce por el arrastre de material producto del escurrimiento superficial [17], ya sea inducida por la intensificación de fenómenos como la Niña, que incrementan la carga de SDT, o por la materia orgánica (MO) y la hojarasca entregadas al río. Así, la acentuación de actividades antropogénicas puntuales, como son el pastoreo cerca de las riveras, la aplicación de abonos orgánicos, fertilizantes y las descargas puntuales pueden alterar el flujo de escorrentía y, por ende, la carga de nutrientes y sedimentos que llegan al río e incrementan el carbono orgánico (CO) que puede ser exportado [6].

El COT del año 2009 es significativamente superior al del año 2010 y 2011; igual comportamiento tienen la alcalinidad y el $\mathrm{pH}$. El incremento de alcalinidad y pH en este año se puede atribuir al lavado de bases intercambiables de suelo producto del 


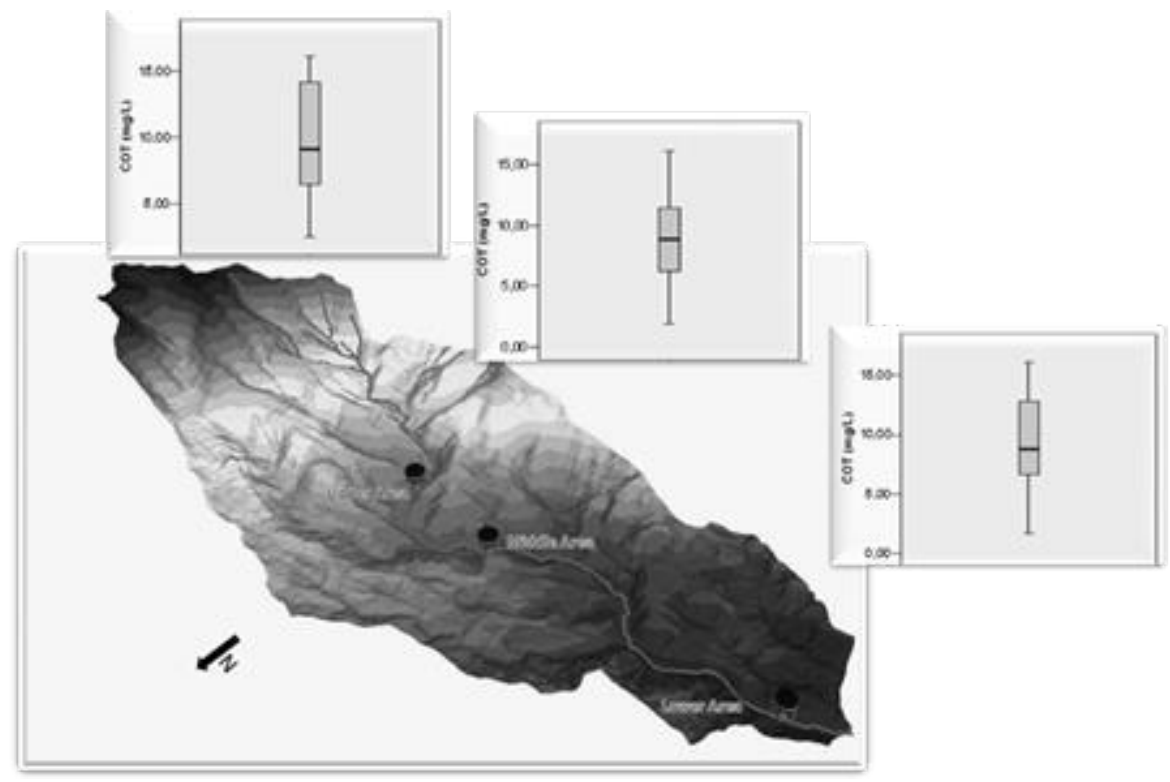

Figura 2. Contenido de COT en el río Las Piedras Q2= mediana, $Q 1=$ mínimo, Q3= máximo.

Fuente: elaboración propia

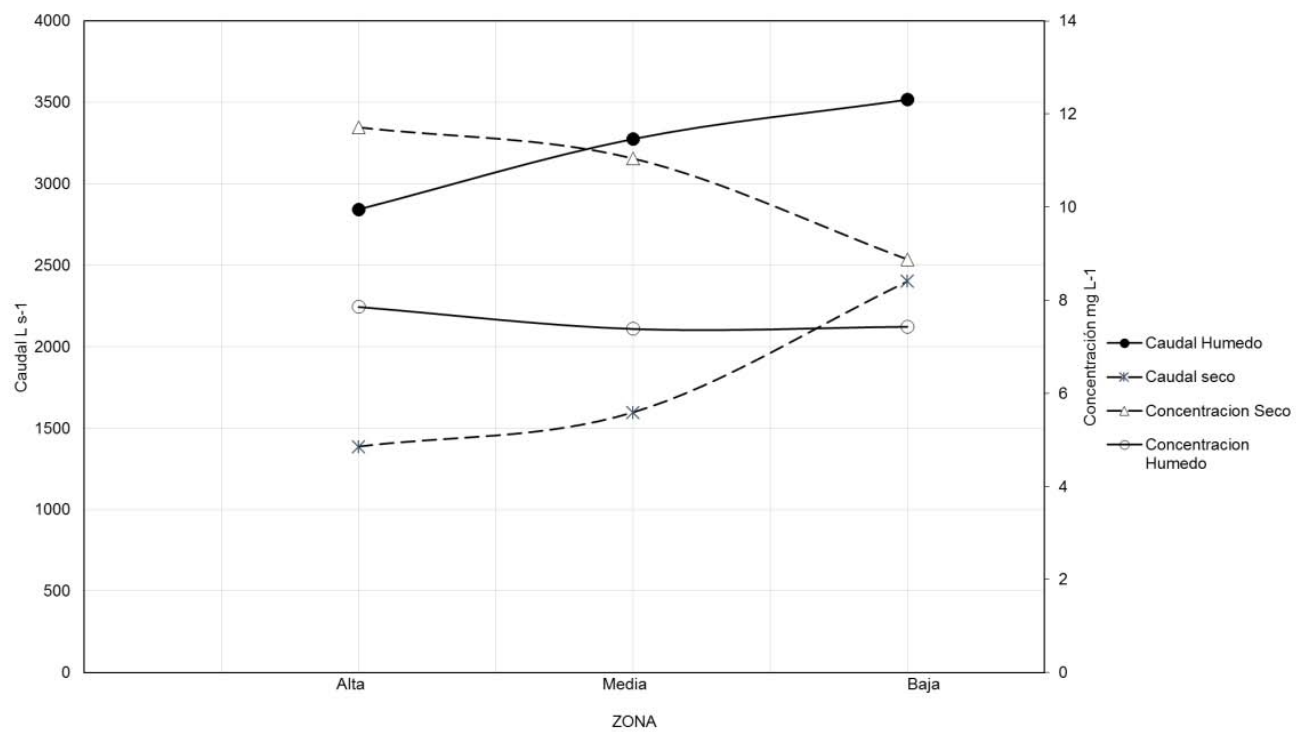

Figura 3. Variación estacional del caudal y la concentración de carbono.

Fuente: elaboración propia 

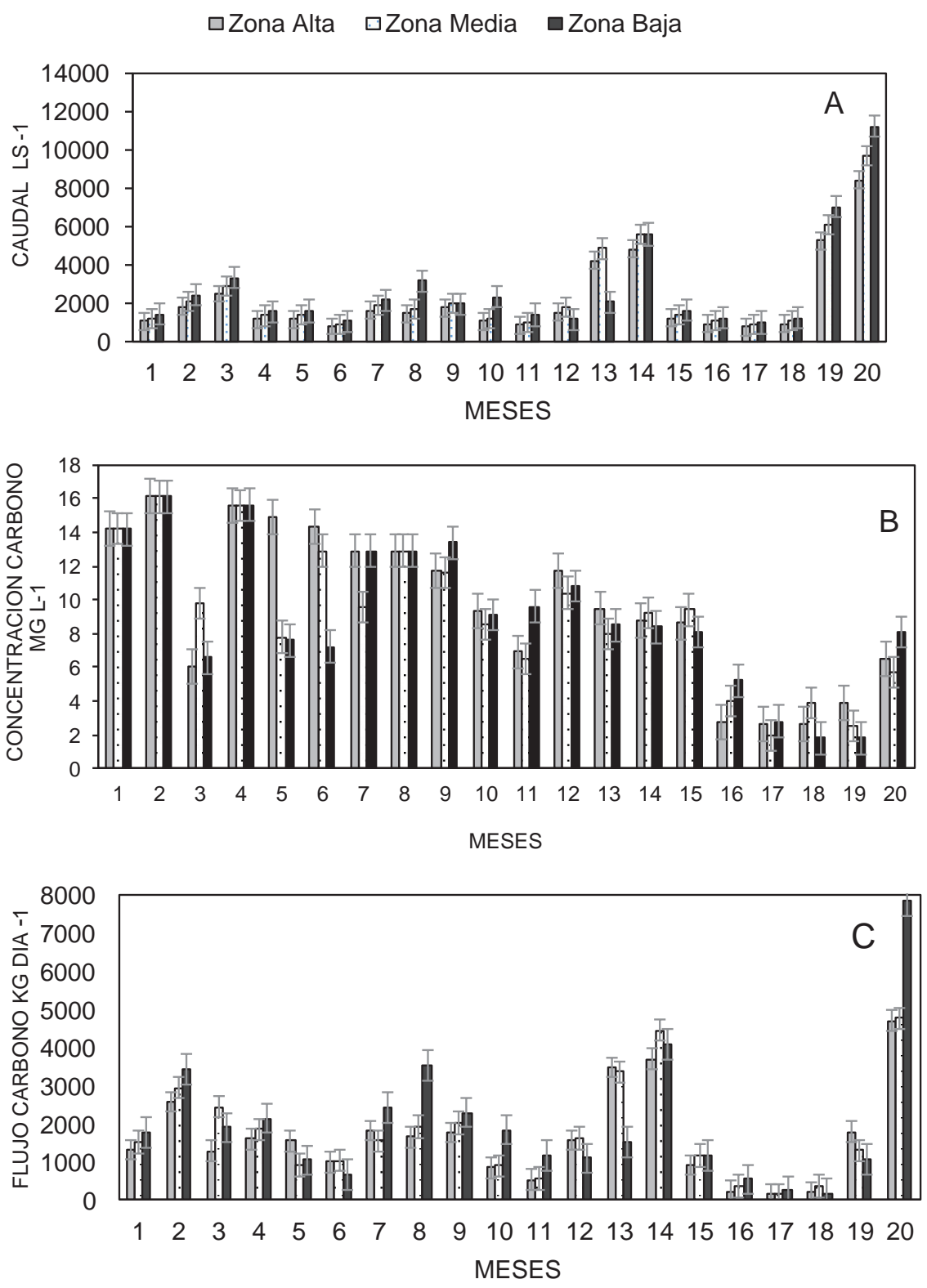

Figura 4. Variación temporal. a) Flujo de carbono b) Caudal C) Concentración de carbono.

Fuente: elaboración propia

encalamiento de suelos, y además, a la disolución de bases de Ca y Mg en forma de bicarbonatos provenientes de la disolución de $\mathrm{CO}_{2}$ en las aguas superficiales, aumentando así el contenido de $\mathrm{CO}_{3}{ }^{2-}$ totales, efecto producido por la ley de Henry (reacciones 
1, 2, 3 ) [18], hipótesis soportada por la correlación positiva y altamente significativa entre COT y $\mathrm{pH}\left(\mathrm{r}_{\mathrm{s}}=0,379 * *\right)$.

$$
\begin{aligned}
& \mathrm{CO}_{2}+\mathrm{H}_{2} \mathrm{O} \rightarrow \mathrm{H}_{2} \mathrm{CO}_{3} \\
& \mathrm{H}_{2} \mathrm{CO}_{3} \rightarrow \mathrm{HCO}_{3}^{-}+\mathrm{H}^{+} \\
& \mathrm{HCO}_{3}^{-}+\mathrm{CaCO}_{3} \rightarrow \mathrm{Ca}\left(\mathrm{HCO}_{3}\right)^{2}
\end{aligned}
$$

El incremento en la disolución del $\mathrm{CO}_{2}$ en el rio produce incremento de $\mathrm{pH}$ y, recíprocamente, disminuyen el OD, demostrado por la correlación negativa y altamente significativa entre OD y $\mathrm{pH}\left(\mathrm{r}_{\mathrm{s}}=-0,335^{* *}\right)$ lo que revela que el cauce del río Las Piedras captura en cierto grado $\mathrm{CO}_{2}$ disponible por efecto de disolución. La correlación positiva y altamente significativa de alcalinidad con SDT $\left(\mathrm{r}_{\mathrm{s}}=0,436^{* *}\right)$ confirma la presencia de carbonatos y bicarbonatos disueltos, e indica disolución $\mathrm{CO}_{2}$ en estas aguas superficiales que, a su vez, incrementa la CE; debido a estas y otras funciones, los flujos, fuentes, y los mecanismos de CO, el transporte y la transformación son algunos de los temas de actualidad más importantes de la biogeoquímica marina y de agua dulce [19].

El aporte de compuestos orgánicos tales como ácidos fúlvicos provenientes de arrastre o lixiviación del suelo, producto de intervenciones antropogénicas, principalmente de la actividad agropecuaria, y cambio de uso del suelo, están alterando la MO libre susceptible a fuerte mineralización [20], producen efectos adversos en la calidad del agua por la disminución del OD, y dan lugar a la degradación del ecosistema. De esta forma, el carbono orgánico puede entrar en el sistema a través de la precipitación, la lixiviación y la descomposición [20]. Resultados similares reportaron Rixen et al. [21] quienes muestran que el aumento de las entradas de carbono orgánico reducen las concentraciones de oxígeno. La MO, disuelta en forma de ácidos fúlvicos, podría quelar las bases $\mathrm{Ca}$ y $\mathrm{Mg}$, y ocasionar disminución en la alcalinidad, lo que explica la correlación negativa entre el COT y la alcalinidad $\left(\mathrm{r}_{\mathrm{s}}=-0,153\right)$. Sin embargo, en este caso predomina la formación de bicarbonatos que incrementan el valor de alcalinidad y $\mathrm{pH}$, demostrado por el alto coeficiente de correlación de Spearman.

El incremento significante en el flujo fluvial del COT podría no solo significar la pérdida del valioso almacenamiento de carbono terrestre, sino que daría lugar al aumento en los niveles de degradación del ecosistema acuático por la introducción de metales pesados y polución orgánica en el recurso hídrico, que podrían afectar la penetración de luz y producir compuestos cancerígenos trihalometanos durante el tratamiento de agua con cloro, además de afectar la calidad de la misma en términos de olor, color, sabor, estética, el equilibrio ácido-base y la complexión con metales. 
La temperatura del agua del río, además, tiene influencia en el COT y $\mathrm{pH}$ de acuerdo con las correlaciones positivas y altamente significativas $\left(\mathrm{r}_{\mathrm{s}}=0,207^{* *}\right.$ y $\left.\mathrm{r}_{\mathrm{s}}=0,199^{* *}\right)$; un incremento en la temperatura aumenta la disolución del $\mathrm{CO}_{2}$ y de compuestos orgánicos.

El incremento en el contenido de N-orgánico e N-inorgánico disminuye el OD, probablemente por efectos de nitrificación consumiendo el OD, como se explica en la relación negativa y significativa entre estos dos parámetros $\left(\mathrm{r}_{\mathrm{s}}=-0,226^{* *}\right)$. La relación negativa entre $\mathrm{N}$ y precipitación $\left(\mathrm{r}_{\mathrm{s}}=-0,338^{* *}\right)$ expone que las lluvias no aportan concentraciones importantes de compuestos nitrogenados a estas aguas superficiales.

El N no sufre cambios significativos en el transcurso del tiempo analizado, aun cuando el COT disminuye, por lo que se puede inferir que hay aporte de $\mathrm{N}$ inorgánico $\left(\mathrm{NO}_{3}{ }^{-}\right.$y $\left.\mathrm{NH}_{4}{ }^{+}\right)$provenientes de las actividades agropecuarias mencionadas. También cabe la posibilidad de una fijación atmosférica de $\mathrm{N}$ por microorganismos no simbióticos, capaces de difundir $\mathrm{N}_{2}$ gaseoso en sus estructuras y convertirlo en $\mathrm{N}$ mineral $\left(\mathrm{NO}_{3}{ }^{-}\right.$y $\left.\mathrm{NH}_{4}{ }^{+}\right)$; sin embargo, los compuestos nitrogenados presentes parcialmente son compuestos orgánicos.

De las correlaciones negativas entre SDT $\left(r_{\mathrm{s}}=-0,636^{* *}\right)$, alcalinidad $\left(\mathrm{r}_{\mathrm{s}}=-0,247^{* *}\right)$ y CE $\left(\mathrm{r}_{\mathrm{s}}=-0,621^{* *}\right)$, con la precipitación se deduce que las lluvias no aportan contaminantes inorgánicos a estas aguas superficiales.

\section{CONCLUSIONES}

El flujo del COT en la cuenca del río Las Piedras depende principalmente de la geomorfología típica de la cuenca y del incremento del caudal asociado a la cantidad de precipitación. El 99,6 \% de la cuenca transporta en promedio un flujo de 2003,5 kg día ${ }^{-1}$ de COT. La relación entre el COT y otras variables físico-químicas que determinan la calidad del agua revela que existe disolución de $\mathrm{CO}_{2}$ en el cauce principal del río Las Piedras.

La intensificación de actividades antropogénicas en áreas de influencia directa al rio, principalmente asociadas a preparación de tierras para cultivos y descargas residuales, altera la calidad del recurso hídrico, y afecta el transporte actual de COT y la disolución de $\mathrm{CO}_{2}$ en el río. La modificación del ciclo natural del carbono puede deteriorar servicios ecosistémicos vitales como el suministro de agua con calidad y la captura de carbono, repercutiendo en la integridad de la población humana local y de la ciudad de Popayán. 


\section{AGRADECIMIENTOS}

Los autores agradecen al Departamento Administrativo de Ciencias, Tecnología e Innovación - Colciencias por la financiación del presente proyecto. También agradecen al Grupo de Estudios Ambientales (GEA) y a la Vicerrectoría de Investigaciones de la Universidad del Cauca por el apoyo en el trabajo.

\section{REFERENCIAS}

[1] T.-H. Huang et al., "Fluvial carbon fluxes in tropical rivers," Current Opinion in Environmental Sustainability vol. 4, pp. 162-169, 2012.

[2] R. T. Barnes, y P. A. Raymond, "The contribution of agricultural and urban activities to inorganic carbon fluxes within temperate watersheds", Chemical Geology, vol. 266, pp. 318$327,2009$.

[3] R. O. Carey et al., "Nutrient discharges to Biscayne Bay, Florida: Trends, loads, and a pollutant index", Science of The Total Environment, vol. 409, n. ${ }^{\circ}$ 3, pp. 530-539, 2011.

[4] Corporación Autónoma Regional del Cauca, "Plan de ordenación y manejo de la subcuenca hidrográfica del río Las Piedras”, 2006.

[5] X. Li et al., "Partitioning soil respiration and assessing the carbon balance in a Setaria italica (L.) Beauv. Cropland on the Loess Plateau, Northern China," Soil Biology and Biochemistry, vol. 42, n. ${ }^{\circ}$ 2, pp. 337-346, 2010.

[6] K. S. Veum et al., "Runoff and dissolved organic carbon loss from a paired-watershed study of three adjacent agricultural Watersheds," Agriculture, Ecosystems \& Environment, vol. 130, n. $3-4$, pp. 115-122, 2009.

[7] A. M. Hook, y J. A. Yeakley, "Stormflow Dynamics of Dissolved Organic Carbon and Total Dissolved Nitrogen in a Small Urban Watershed”, Biogeochemistry, vol. 75, pp. 409-431, 2005.

[8] A.-M. Aucour et al., "Fluxes and sources of particulate organic carbon in the Ganga-Brahmaputra river system”, Global Biogeochem. Cycles, vol. 20, n. 2, p. 12, 2006.

[9] M. I. Bird et al., "A preliminary estimate of organic carbon transport by the Ayeyarwady (Irrawaddy) and Thanlwin (Salween) Rivers of Myanmar", Quaternary International vol. 186 pp. 113-122, 2008.

[10] E. H. Stanley et al., "Contemporary changes in dissolved organic carbon (DOC) in humandominated rivers: is there a role for DOC management?", Freshwater Biology 2011.

[11] IGAC, Estudio general de suelos y zonificación de tierras departamento del Cauca, p.^pp. 556, Bogota: Instituto Geográfico Agustín Codazzi, 2009, p.

[12] APHA et al., Standar methods for the examintation of water an wastewater Washington, D. C.: American Public Health Association, 2005, p.

[13] GEA, "Modelamiento climático, patrones de cambio y sus efectos en ecosistemas agrícolas altoandinos, identificando medidas de adaptación y mitigación para la planificación. MACACEA", Ministerio de Agricultura y Desarrollo Rural 2012, p. 84. 
[14] O. Martins, y L. probst, "Biochemistry of major African rivers: Carbon and Mineral Transport”, en Biogeochemistry of Major world Rivers, E. degens, ed., Seattle, USA: SCOPE, 1991.

[15] M. Pełechaty et al., "Spatial and Temporal Variability of TOC Concentrations in a Shallow and Eutrophicated Lake Ecosystem (Lake Jarosławieckie, Wielkopolski National Park, Western Poland)", Polish Journal of Environmental Studies vol. 12, n. ${ }^{\circ}$ 5, pp. 607-611, 2003.

[16] Y. Wu et al., "Biogeochemical behavior of organic carbon in a small tropical river and estuary, Hainan, China”, Continental Shelf Research, 2012.

[17] A. Townsend-Small et al., "Suspended sediments and organic matter in mountain headwaters of the Amazon River: Results from a 1-year time series study in the central Peruvian Andes", Geochimica et Cosmochimica Acta vol. 72, pp. 732-740, 2008.

[18] H. Fassbender, y E. Bomemisza, Química de los suelos con énfasis en suelos de América Latina, Costa Rica: Instituto Interamericano de Cooperación para la Agricultura (IICA), 1987, p.

[19] E. T. Degens et al., ed, "Biogeochemistry of Major World Rivers", Chichester: Wiley, 1991, 323-348. p.

[20] T. Amann et al., "Carbon dynamics in the freshwater part of the Elbe estuary, Germany: Implications of improving water quality”, Estuarine, Coastal and Shelf Science, vol. 107, n. ${ }^{\circ}$ 0, pp. 112-121, 2012.

[21] T. Rixen et al., "Dissolved oxygen and its response to eutrophication in a tropical black water river", Journal of Environmental Management, vol. 91, n. ${ }^{\circ}$ 8, pp. 1730-1737, 2010. 